\title{
HLA Class IC Histocompatibility Antigen Measurement
}

National Cancer Institute

\section{Source}

National Cancer Institute. HLA Class IC Histocompatibility Antigen Measurement. NCI

Thesaurus. Code C154748.

The determination of the amount of HLA class IC histocompatibility antigen present in a sample. 